\title{
Single Local Interneurons in the Locust Make Central Synapses with Different Properties of Transmitter Release on Distinct Postsynaptic Neurons
}

\author{
Gilles Laurent ${ }^{1}$ and Anand Sivaramakrishnan² \\ ${ }^{1}$ California Institute of Technology, Biology Division, Computation and Neural Systems Program, Pasadena, California \\ 91125, ${ }^{2}$ The Observatories of the Carnegie Institution of Washington, Pasadena, California 91101, and Department of \\ Physiology and Biophysics, New York University Medical Center, New York, New York 10016
}

Quantal analysis has been applied to the inhibitory synapses made by single spiking local interneurons onto several nonspiking local interneurons (and motorneurons) in the locust CNS. Transmission at these synapses appears to be mediated by GABA. The apparent reversal potential of the IPSP and inhibitory postsynaptic current were -80 to $-85 \mathrm{mV}$, a value similar to that of the potential evoked by pressureapplied GABA. This reversal potential was 25-30 mV more negative than the resting potential of the nonspiking interneurons in the experimental conditions. The statistical properties of release at these synapses were studied by recording simultaneously from pre- and postsynaptic interneurons with intracellular electrodes. The distribution of postsynaptic potential amplitudes could be described by a simple binomial model, implying uniformity of binomial $p$ (probability of release at a single release site) for each synapse. The mean quantal amplitude was $290 \pm 110 \mu \mathrm{V}$, and the mean quantal content $m$ of the IPSPs was $6.25 \pm 2.83$. The mean values of binomial $n$ (average size of the releasable pool) and $p$ were $13.11 \pm 2.8$ and $0.45 \pm 0.16$, respectively. Numerical simulations of statistical experiments were performed to test whether the IPSP amplitude distribution histograms might be misleadingly indicative of quantal release. These simulations showed that such a hypothesis was very unlikely. When a spiking local interneuron was impaled, several of its target interneurons could sometimes be successively sampled. Quantal analysis was then performed with the different IPSPS evoked, in identical conditions, by a same presynaptic interneuron, and the quantal parameters were compared between the synapses. It was found that binomials $n$ and $p$ and their product $m$ generally differed between the synapses made by a given spiking interneuron onto different target neurons. These results show that quantal contents can vary for the many synapses made centrally by one interneuron,

\footnotetext{
Received Sept. 26, 1991; revised Jan. 16, 1992; accepted Jan. 21, 1992.

We are grateful to Drs. M. Brodwick, M. Burrows, S. Sivaramakrishnan, R. W. Tsien, and two anonymous referees for their many helpful comments on this work which was supported by grants from NIH (NS29194-01), the Human Frontier Science Program, and the ONR and by Searle, Alfred P. Sloan, and McKnight scholarships to G.L.

Correspondence should be addressed to Gilles Laurent, California Institute of Technology, Computation and Neural Systems Program, Biology Division, 139 74, Pasadena, CA 91125.

Copyright (C) 1992 Society for Neuroscience $0270-6474 / 92 / 122370-11 \$ 05.00 / 0$
}

and suggest that this variability may arise from differences in release probabilities between the sites associated with different synapses.

Central neurons typically synapse with many other neurons. In mammals, for example, a neuron in the inferior olivary nucleus may form up to 10 climbing fibers, which cach contact a different Purkinje cell in the cerebellum (Llinás and Walton, 1990), and a thalamic afferent may connect with several thousand cortical neurons (Freund et al., 1985). In invertebrates, a single proprioceptive afferent can diverge to several motorneurons and local interneurons (Burrows, 1987a; Laurent and Hustert, 1988), and a single nonspiking local interneuron can diverge to several motomeurons within a pool, or in different pools (Burrows and Siegler, 1978; Burrows, 1980). A knowledge of the relative influence that a neuron has on its many central targets and of the mechanisms by which variability of release properties might come about is thus critical for an understanding of network integration. The differences in the release properties at the synapses made centrally by a single neuron onto different targets have, however, apparently not yet been investigated.

Quantal analysis of neurotransmission at chemical synapseswhich describes the quantization of postsynaptic potentials (PSPs) or currents (PSCs) and their variability - has been most successful at the neuromuscular junction (DelCalstillo and Katz, 1954; Boyd and Martin, 1956), where it was first developed. Such analysis, however, has been more difficult to apply to connections in the CNS, and there is still much contention as to which statistics, if any (e.g., Poisson, simple or compound binomial), best describe transmission at these synapses (Walmsley et al., 1987; Faber and Korn, 1988; Edwards et al., 1990; Larkman et al., 1990; Redman, 1990). Quantal analysis was performed here, and Poisson and simple binomial models used, to study the release properties of synapses between local interneurons in the insect CNS. Typically, a single presynaptic interneuron was impaled and several targets were successively sampled. Whether differences in release properties existed between these different synapses was then examined.

Experiments were designed using the central synapses made from uniquely identifiable spiking to nonspiking local interneurons, which underlie the execution of tactile and proprioceptive reflexes of the legs in the locust (Burrows, 1987b). Spiking local interneurons (Fig. $1 A$ ) receive direct inputs from leg mechanoreceptors (Siegler and Burrows, 1983; Burrows, 1987a) and make inhibitory connections with other spiking local interneu- 

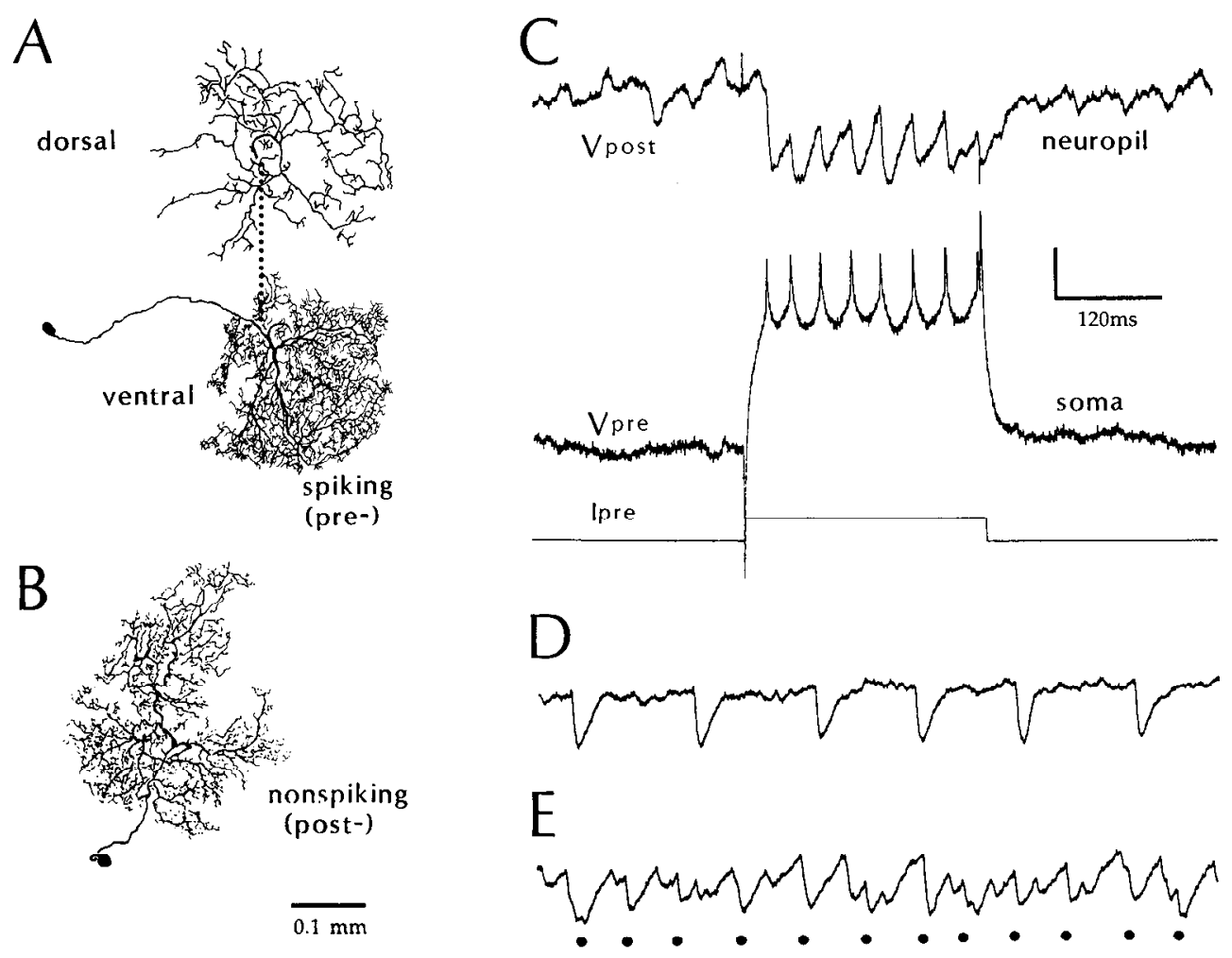

Figure 1. Fluctuations of IPSP amplitudes at central synapses between spiking and nonspiking local interneurons can be studied using paired intracellular recordings in vivo. $A$, Dorsal view of a spiking local interneuron stained by intracellular injection of cobalt. Neurons in this population have a small soma, a long primary neurite that lacks active properties, a dense ventral field of branches bearing the majority of input synapses, and a sparse field of dorsal and varicose branches, where most outputs are made. Dorsal and ventral fields are connected by an unbranched "linking" process represented here by a broken line. Recordings were made from the soma. $B$, A nonspiking local interneuron. Input and output synapses are intermingled on its branches (Watson and Burrows, 1988). Recordings were made from a neurite rather close to the synaptic sites. $C$, Paired recording from two connected neurons showing the 1:1 correlation between action potentials in the spiking interneuron and IPSPs in the nonspiking interneuron. Action potentials in the spiking interneuron are only a few millivolts in amplitude because of the low-pass filtering effect of the long and inactive primary neurite separating the soma from the integrative and active membrane. $D$ and $E$, Traces from two nonspiking interneurons showing variations in background synaptic noise. $D$, Interneuron in which the prominent IPSPs are caused by a single presynaptic spiking local interneuron. $E$, Another interneuron, in which IPSPs evoked by spikes in one recorded spiking interneuron (record of the presynaptic neuron not shown, but IPSPs indicated by dots) sum with other PSPs evoked by other neurons. Calibration: $C, 1 \mathrm{mV}$ (top), $2 \mathrm{mV}$ (middle), $1 \mathrm{nA}$ (bottom); $D$ and $E, 1 \mathrm{mV}$, time same as in $C$.

rons (Nagayama and Burrows, 1990), nonspiking local interneurons (Burrows, 1987b), motorneurons (Burrows and Siegler, 1982), and intersegmental interneurons (Laurent, 1987). These output connections appear to be made mostly from the varicosities in a sparse field of branches in the dorsal neuropil of a segmental ganglion (Fig. 1A) (Watson and Burrows, 1985). The nonspiking local interneurons (Fig. $1 B$ ) are premotor interneurons, and have intermingled input and output synapses (Watson and Burrows, 1988). A single spiking interneuron can make divergent conncctions to scveral nonspiking intcrncurons (Burrows, 1987b). Similarly, a nonspiking interneuron generally receives convergent inputs from several spiking interneurons (Burrows, 1987b), making the attribution of spontaneous PSPs to any specific presynaptic neuron impossible. For this reason, simultaneous intracellular recordings were made from presynaptic spiking local interneurons in their soma, which is distant from their outpul synapses, and from postsynaptic nonspiking local interneurons in one of their dendrites, close to the input synapses (Fig. 1 $A-C$ ).

\section{Materials and Methods}

Morphology. The local interneurons in Figure 1 were stained in two different preparations, by intracellular injection of $6 \%$ cobalt hexam- mine chloride (Pitman et al., 1972), intensified with silver (Timm's intensification method; Bacon and Altman, 1977), and drawn in wholemount with a camera lucida. They are viewed dorsally. The dorsal and ventral fields of the spiking interneuron in Figure $1 A$ have been artificially separated during drawing. They are joined by a ventrodorsal "linking" process devoid of side branches, represented here by a broken line. The great majority of output synapses in spiking local interneurons are found in the dorsal, sparse, and varicose branches that overlap with the neurites of motor neurons and other local and intersegmental interneurons. Some, however, occur in the dense field of branches in the ventral neuropil, where their role is so far unknown (Watson and Burrows, 1985). The neurons used in this study were identificd as members of a class and not as individuals, since all the physiological and morphological tests could not be performed with all experiments.

Electrophysiology. Adult animals (Schistocerca americana) of both sexes were obtained from a crowded lab colony. The recordings were made from the isolated thoracic nervous system preparation described in Laurent (1990). Recordings from the nonspiking interneurons were made from a neurite rather than the soma. The larger branches of nonspiking local interneurons can be $5-8 \mu \mathrm{m}$ in diameter and thus sometimes allow very stable recording. The preparation was continually superfused with aerated locust saline ( $140 \mathrm{~mm} \mathrm{NaCl}, 10 \mathrm{~mm} \mathrm{KCl}, 4 \mathrm{~mm}$ $\mathrm{CaCl}_{2}, 4 \mathrm{mM} \mathrm{NaHCO}_{3}, 6 \mathrm{~mm} \mathrm{NaH} \mathrm{PO}_{4}$, and $34 \mathrm{gm} /$ liter sucrose to adjust osmolarity; $\mathrm{pH} 7.0$ at $23^{\circ} \mathrm{C}$ ). Intracellular recordings for the physiological experiments were made at a room temperature of $21-23^{\circ} \mathrm{C}$, with thick-walled glass micropipettes filled with $3 \mathrm{M} K$-acetate with DC resistance of 40-80 M $\Omega$. Signals from the two neurons recorded simultaneously were amplified with two Axoclamp-2A (Axon Instruments 
Inc.) amplifiers to reduce capacitive coupling between channels, and precautions were taken to reduce stray recording noise to a minimum. This often included coating the microelectrodes close to the tip and reducing the saline level (Laurent, 1990). For the single-electrode current- and voltage-clamp recordings, the headstage voltage was monitored on a separate oscilloscope, to ensurc complete settling at the end of each switching cycle. Switching rates of $5-6 \mathrm{kHz}$ were routinely obtained, allowing nearly complete electrode voltage decay before sampling. Data were filtered at 0.3 or, more generally, $1 \mathrm{kHz}$, recorded on an eight-channel Digital Audio Tape recorder, using a sampling rate of 5 or $10 \mathrm{kHz}$ per channel, and analyzed on a microcomputer after further amplification and digitization at $10 \mathrm{kHz}$. Signal averaging of voltage waveform was performed with a digital oscilloscope or the microcomputer. For the quantal analysis, both pre- and postsynaptic neurons were recorded in bridge mode, to reduce noise levels. GABA and muscimol were dissolved in saline and pressure injected using a pneumatic "picopump" (World Precision Instruments Inc.). Chemicals were obtained from Sigma Chemical Co. Direct connections between spiking and nonspiking interneurons were found in $10-15 \%$ of recorded pairs of local intcrncurons. Finding several targets to a single spiking local interneuron in a single experiment was therefore very rare, and the successful experiments on which the statistical analysis was carried out are based on recordings from 46 animals. The experiments on the reversal potential of the inhibitory PSC/P and the response to GABA were performed on 16 animals.

Data collection and analysis. Large numbers of IPSPs were evoked at constant frequencies chosen between 1 and $7 \mathrm{~Hz}$. If several pairs of connected interneurons were obtained successively with a same presynaptic spiking interneuron, all IPSPs recorded in the successively impaled postsynaptic neurons were evoked at the same frequency (e.g., $2 \mathrm{~Hz}$ ), so that release properties at the different synapses could be compared. Impalement of a presynaptic spiking local interneuron could very rarely be maintained for much more than $30 \mathrm{~min}$ to $1 \mathrm{hr}$, so that no more than a few minutes elapsed between recording sessions of several targets to a single presynaptic interneuron. Spikes in the presyraptic interneuron were evoked by short depolarizing pulses at the selected frequency to evoke single spikes, while holding the interneuron silent between pulses with DC hyperpolarizing current. Alternatively, the interneuron was held with DC current at such a membrane potential that it fired regularly at the selected frequency. Successive IPSPs were then sampled and their peak amplitude measured. The time-to-peak voltage was determined from an averaged template of 32-64 traces. All these experiments were performed in normal $\mathrm{Ca}^{2+}$ saline. IPSPs rather than IPSCs were collected because the switching frequencies allowed by the discontinued-clamp amplifier, the electrodes, and the nonspiking interneurons added considerable noise to the signal. IPSPs were thus collected in bridge mode. The average IPSP and quantal IPSP amplitudes were $7.7 \%$ and $1.2 \%$ of the synaptic driving force, respectively (see Results), thus limiting the extent of nonlinear summation. This was confirmed by the overall regular spacing of peaks in the IPSP amplitude distribution histograms, and no correction method was employed here.

Amplitude histograms were constructed from stretches of consecutive IPSPs in stationary conditions. Since a nonspiking interneuron receives inputs from many presynaptic neurons, the attribution of spontaneous potentials to a specific presynaptic neuron was impossible. The putative quantal amplitude $q$ (and variance $\sigma_{q}^{2}$, see below) could therefore not be determined from asynchronously released miniature IPSPs. Also, because the experiments were conducted in normal $\mathrm{Ca}^{2+}$ saline and because failures were in such conditions usually very rare, the mean quantal content $m$ of each connection could not reliably be estimated by using the "method of failures." Rather, $q$ was estimated from the averaged interval $d V$ between peaks in the amplitude histogram, and $m$ was first estimated indirectly as $I P S P / q$, where $I P S P$ is the average IPSP amplitude. After the binomial amplitude distribution had been constructed, the product $m^{\prime}=n p$ of the so-obtained best-fitting binomials $n$ and $p$ was compared to the value of $m$ estimated indirectly. Finally, for those connections best fitted by the binomial frequency distribution (9 out of 10$), m$ was estimated using the coefficient of variation of the IPSPS $(\mathrm{CV})$ as

$$
m^{\prime \prime}=\left\{1-I P S P / I P S P_{\max }\right\} \mathrm{CV}^{-2},
$$

where $I P S P_{\max }$ is the maximum IPSP amplitude in the sample. $m^{\prime \prime}$ was then compared to $m$. Each peak was assumed to be fitted by a Gaussian distribution with a variance $V_{x}=x V_{q}=x \sigma_{q}^{2}$, where $V_{q}$ is the quantal variance and $x$ the number of quantal units. This variance was assumed to arise principally from quantal IPSP amplitude fluctuations because the signal-to-noise ratio was usually high (see Figs. 1, 4-6). $\sigma_{q}$ remained a free parameter, and its value was obtained from the best-fitting theoretical distribution. The Gaussians were then added together. The two theoretical frequency distributions tested were (1) a simple binomial distribution, which assumes uniformity and stationarity of $p$ (see Results), and (2) a Poisson distribution, which only depends on the determination of $m$ (McLachlan, 1978). Comparisons of the observed and theoretical distributions were made using the $\chi^{2}$ test with 2 (Poisson) or 3 (binomial) degrees of freedom less than the number of bins (McLachlan, 1978). The statistical analysis and curve fitting were performed on a Macintosh IIcx using Mathematica@. The calculations of our numerical simulation experiments were performed on a Sun SPARCstation IPX, using the random number generator RANDOM( $)$ and a state length of 32 four-byte words.

\section{Results}

\section{The postsynaptic response}

A barrage of action potentials evoked by direct current injection into a spiking interneuron evokes 1:1 IPSPs in a nonspiking interneuron, superimposed on a background of PSPs caused by other converging neurons (Fig. $1 C, E$ ). The connection is monosynaptic and IPSPs appear within a consistent delay of 1-1.5 msec (Burrows, 1987b). No known polysynaptic pathways contribute to the postsynaptic response, and no reciprocal connection has been shown to exist. To ascertain the risk of nonlinear summation of quantal IPSPs at the resting potential of the nonspiking interneurons, the driving force of the IPSPS needed to be established by measuring their reversal potential. The reversal potentials of the IPSP and of the IPSC were studied by recording intracellularly from pairs of connected interneurons, and by holding the postsynaptic membrane at various potentials, using the discontinuous current-clamp or voltage-clamp technique, while the presynaptic neuron was made to spike (Fig. 2). The recording in Figure $2 A$ shows that the IPSP apparently reversed around $-80 \mathrm{mV}$ (mean, $-83 \mathrm{mV} ; N=6$ interneurons), and became depolarizing at more negative potentials. Using the single-electrode voltage-clamp technique, the apparent reversal potential of the IPSC was found to be between -80 and -90 $\mathrm{mV}$ (mean, $-85 \mathrm{mV} ; N=4$ interneurons) (Fig. $2 B, C$ ). It is noted that the time constant of decay of the IPSCs did not seem to depend noticeably on the membrane potential at which the nonspiking interneuron was held. This contrasts with the time course of the IPSPs, which strongly depends on membrane potential and resistance (Laurent, 1990). In the present recording conditions, the resting potential of the nonspiking interneurons was about $-57 \mathrm{mV}$, that is, about $25 \mathrm{mV}$ more depolarized than the reversal potential for the IPSC/P. At this resting potential, voltage- and time-dependent outwardly rectifying conductances are at their threshold for activation (Laurent, 1990, 1991). Hence, no effort was made to hold the nonspiking interneurons at more depolarized potentials to increase the driving force of the IPSC/Ps. Such manipulation of membrane potential would have decreased the input resistance of the nonspiking interneurons and introduced undesired nonlinearities for the quantal analysis.

Because spiking local interneurons in the population studied here show GABA-like immunoreactivity (Watson and Burrows, 1987), the response of the postsynaptic nonspiking interneurons to pressure-applied GABA was studied. A nonspiking interneuron was impaled and short negative current pulses were applied at regular intervals through the microelectrode, to monitor for possible changes in input conductance caused by the release of 
A

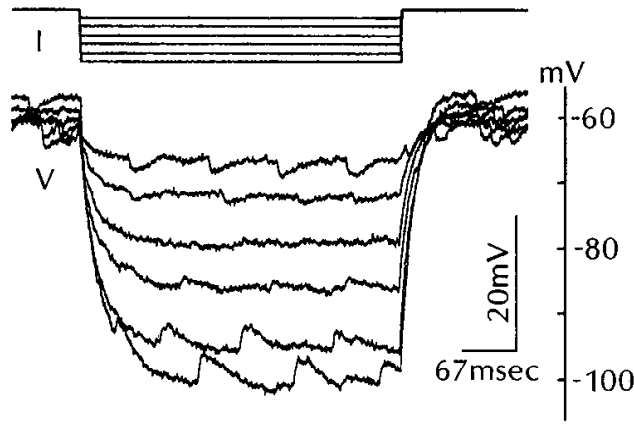

B

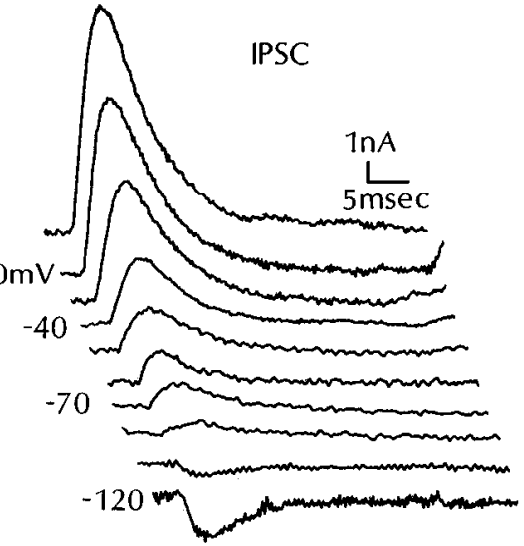

C

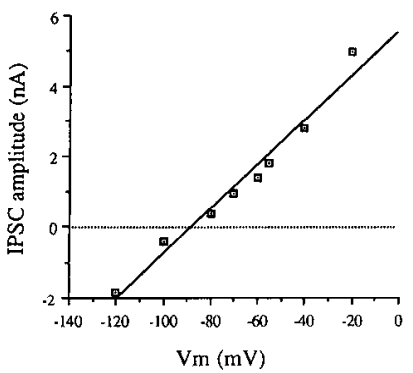

Figure 2. Reversal potential of the IPSP and IPSC in the nonspiking local interneurons. $A$, Current pulses $(I ;-1$ to $-6 \mathrm{nA})$ are injected intracellularly in a nonspiking interneuron to determine the membrane potential $(V)$ at which the IPSPs evoked by a presynaptic spiking local interneuron (not shown) reverse polarity. $B$, Same nonspiking local interneuron, now voltage clamped at potentials between 20 and $-120 \mathrm{mV}$ to determine the reversal potential of the IPSCs. The potentials at which the membrane is held are recorded rather than command potentials. These values, however, are subject to errors, due to the limitations of point clamping and to the uncertainty on the electrotonic distance between the synaptic and recording sites. Each IPSC is the average of 8-32 sweeps. $C$, Current-voltage relationship constructed from data in $B$, showing a reversal potential of about $-90 \mathrm{mV}$ for this IPSC.

GABA. A 50 msec pulse of GABA $(0.5 \mathrm{~m}$ in pipette, $\mathrm{pH} 6.9)$ was applied through a second microelectrode inserted in the neuropil, close to the neuropilar recording site, and the voltage response was examined (Fig. 3). The direct application of GABA consistently evoked a conductance increase (Fig. $3 A-C$ ), and a membrane polarization whose reversal potential was between -80 and $-85 \mathrm{mV}$ (mean, $-81 \mathrm{mV} ; N=4$ interneurons; Fig. $3 A$ ). During the peak of the response, the background of tonic IPSPs caused by spontaneously firing spiking local interneurons disappeared, suggesting that both the IPSPs and the response to GABA reverse at the same membrane potential (Fig. $3 A, B$ ). Finally, the vertebrate $\mathrm{GABA}_{\mathrm{A}}$ receptor agonist muscimol (1
mM in pipette) applied in the same conditions evoked a consistent hyperpolarization from resting potential and a membrane conductance increase.

\section{Statistical properties of release}

Connected pairs were looked for and large numbers (500-3000) of spikes were evoked in the presynaptic neuron at a steady rate of between 1 and $7 \mathrm{~Hz}$. IPSPs recorded in bridge mode rather than IPSCs recorded in switched mode were chosen, to reduce background recording noise. The first 50 spikes and corresponding IPSPs of a series were not sampled, because some small amount of depression was observed with 2 out of the 15 con-

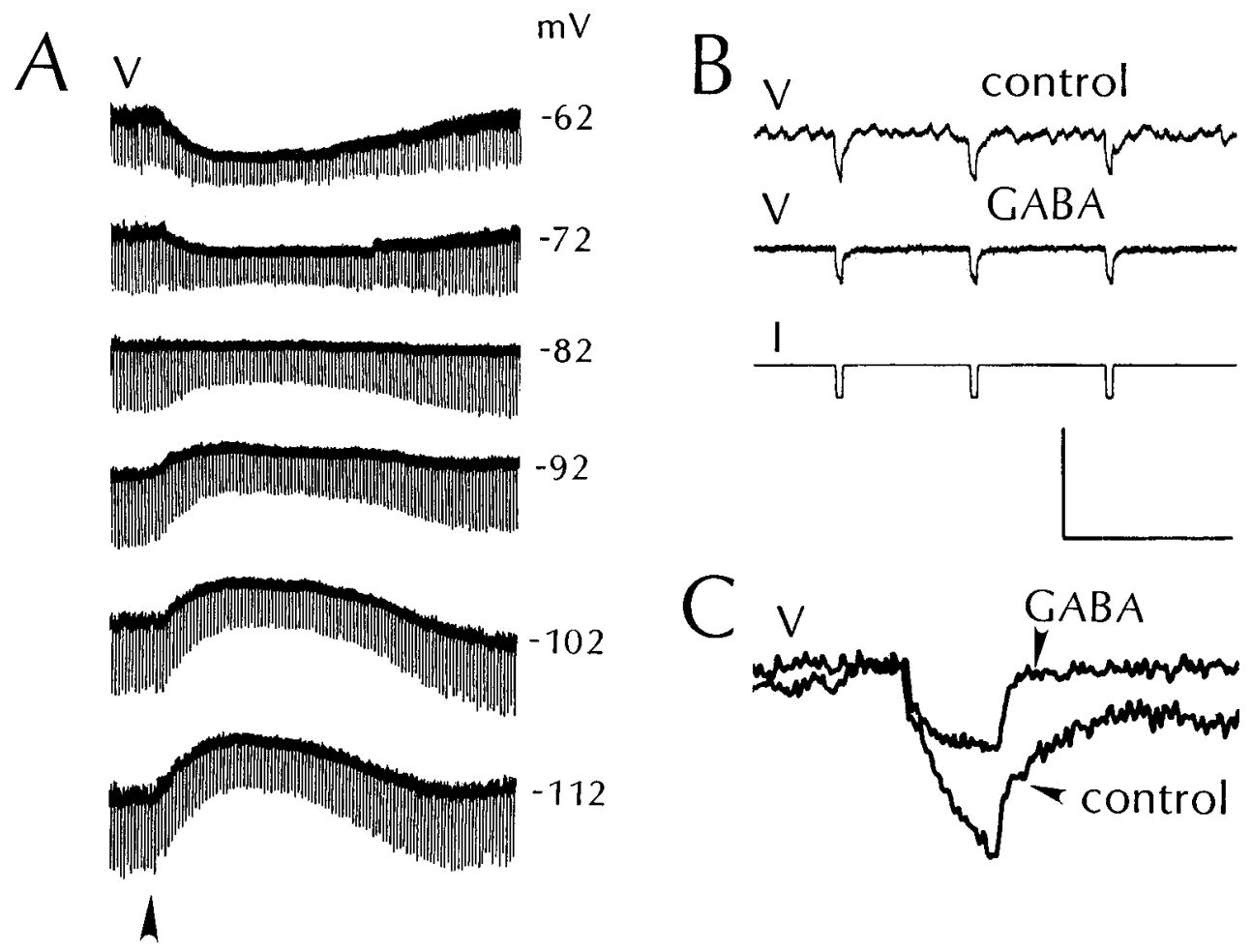

$.5 \mathrm{M} \mathrm{GABA}(50 \mathrm{msec})$

Figure 3. Reversal potential of the response caused by pressure-injected GABA. $A$, A 50 msec ( 25 psi) pulse of $0.5 \mathrm{M}$ GABA injected in the neuropil, close to the recording site, evokes a membrane polarization that reverses between -80 and $-85 \mathrm{mV}$. Note the evoked increased input conductance, monitored by the voltage response to short negative current pulses. Note also that the resting conductance at $-62 \mathrm{mV}$ is less than at more polarized potentials. $B$, The background of IPSPs present before the pulse of GABA (control at $-62 \mathrm{mV}$ ) disappears during the response to GABA (middle trace), suggesting a common reversal potential. $C$, Change in input conductance during the response to GABA, as evidenced by a decreased peak voltage response to constant current pulses. Calibration: vertical, $20 \mathrm{mV}(A, B$ top, $B$ middle), $6 \mathrm{nA}$ ( $B$ bottom), $6 \mathrm{mV}(C)$; horizontal, 10 $\sec (A), 250 \mathrm{msec}(B), 18 \mathrm{msec}(C)$. 
A
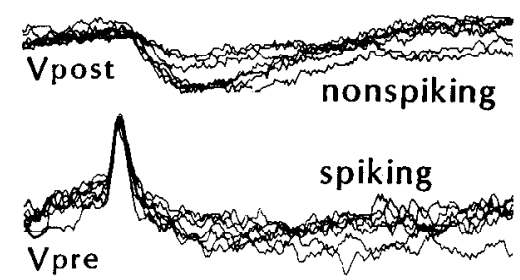

$1 \mathrm{mV}$ (post)

$2 \mathrm{mV}$ (pre)

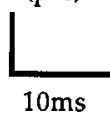

$10 \mathrm{~ms}$

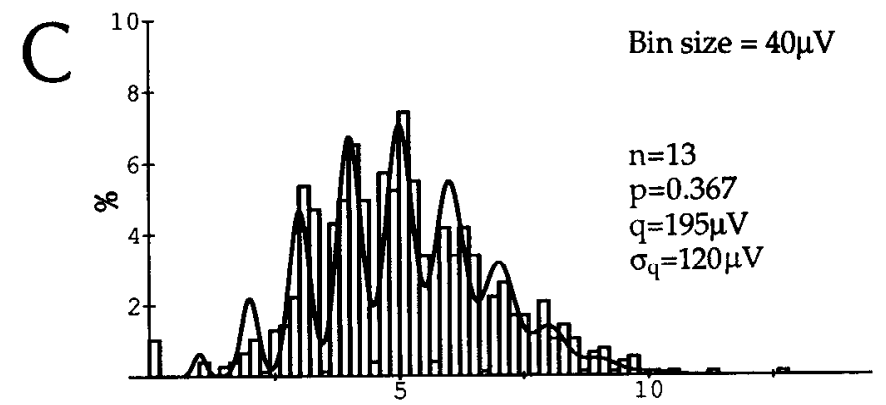

$\mathrm{D}$

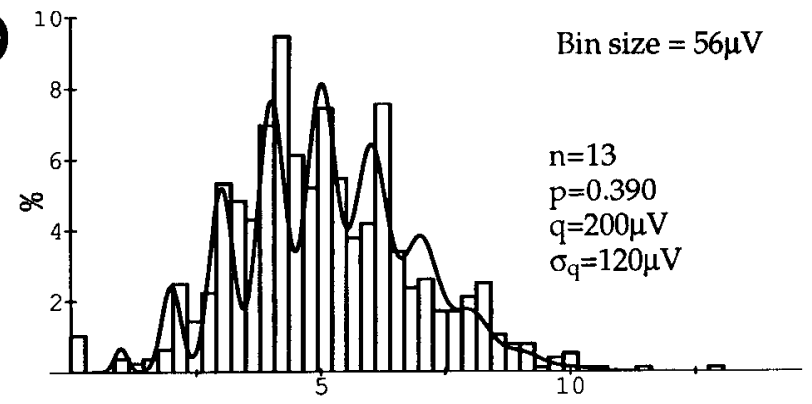

nb of quantal units

Figure 4. Fluctuations of IPSP amplitudes can be described by a simple binomial model. $A$, Superimposed traces of pre- and postsynaptic interneurons showing the fluctuations of IPSP amplitude and the high signal-to-noise ratio. Interval between spikes was 250 msec. $B$, Peak IPSP amplitude histogram built from 768 IPSPs collected successively from the pair in $A$. Peaks in the histogram can be defined and the interval between them used to estimate quantal amplitude. Eight failures were observed. $C$, The same histogram as in $B$, with the best fit using simple binomial statistics and a Gaussian distribution of quantal amplitudes. The variance for each peak was $x$, the number of quantal units, times $\sigma_{q}^{2}$, the variance of one quantum. $D$, Best fit for the same data but now gathered in $56 \mu \mathrm{V}$ bins. The best-fitting parameters are not significantly altered and are thus not dependent on bin size.

nections studied. Continuous stretches (lasting 2-5 min) of such recordings with stable postsynaptic membrane potential were selected from 15 connected pairs of interneurons, and the fluctuations of IPSP amplitudes were displayed on peak amplitude distribution histograms. These pairs include four experiments where a single presynaptic neuron (different each time) was held while two (three times) or three (once) postsynaptic neurons were successively impaled, within a recording period of less than $1 \mathrm{hr}$. Definition of clear peaks in the IPSP amplitude histograms required the additional condition that the synaptic background of the nonspiking interneuron was relatively low. For example, recordings such as that in Figure $1 D$ were rare but optimal, whereas that in Figure $1 E$, in which IPSPs evoked by one spiking interneuron (dots) occurred on top of an already high synaptic background, did not allow a clear definition of quantal peaks, and had to be rejected. Mean IPSP amplitudes recorded from the neurites of the nonspiking interneurons were $1.93 \pm 1.2 \mathrm{mV}$ (mean $\pm \mathrm{SD} ; N=10$ interneurons; range, $0.620-3.71 \mathrm{mV}$; Table 1). The pair in Figure $4, A$ and $B$, shows the statistical fluctuations of IPSP amplitude typical of these connections. When represented on an amplitude distribution histogram, regularly spaced peaks could be visually defined and were taken to indicate quantization of release (Fig. $4 B, C$ ). The tentative mean quantal amplitude $q$ (i.e., the amplitude of the IPSP evoked by release of one putative quantum) was taken as the mean value $d V$ of the intervals between successive peaks in the histogram. The sampling bin size was then changed (Fig. $4 D$ ), and for 10 pairs of interneurons, this did not change $d V$ by more than $\pm 15 \%$. For the synapses made by these selected pairs of interneurons, the quantal amplitude $q$ ranged between 200 and 540 $\mu \mathrm{V}$, with an average of $290 \pm 110 \mu \mathrm{V}$ (Table 1). The mean quantal content $m$ (average number of quanta released by each action potential) was estimated indirectly as the mean IPSP amplitude $/ d V$, and ranged from 2.58 to 10.39 , with an average of $6.25 \pm 2.83$ (Table 1). Stationarity was checked by dividing sets of 600 consecutive IPSPs in groups of 300 , and comparing quantal parameters for each group.

The result that clear and regularly spaced quantal peaks could be defined was encouraging but surprising. The arborizations of spiking and nonspiking local interneurons overlap considerably

Table 1. Description of IPSP amplitude fluctuations and of the binomial parameters providing the best fits to the amplitude distributions for the nine best pairs of neurons

\begin{tabular}{lllcccccc} 
& IPSP $(\mathrm{mV})$ & $q=d V(\mathrm{mV})$ & $m=I P S P / q$ & Binomial $n$ & Binomial $p$ & $m^{\prime}=n p$ & $\sigma_{q}(\mathrm{mV})$ & $m^{\prime \prime}$ \\
\hline Mcan & 1.930 & 0.290 & 6.250 & 13.110 & 0.452 & 6.160 & 0.120 & 5.710 \\
SD & 1.200 & 0.110 & 2.830 & 2.800 & 0.160 & 2.987 & 0.030 \\
Minimum & 0.620 & 0.200 & 2.580 & 9.000 & 0.200 & 2.000 & 0.080 & 1.600 \\
Maximum & 3.710 & 0.540 & 10.390 & 17.000 & 0.680 & 10.200 & 0.190 & 9.900 \\
\hline
\end{tabular}

Note the excellent agreement between the values of $m, m^{\prime}$, and $m^{\prime \prime}$ obtained independently. 
in the neuropil (Siegler and Burrows, 1979, 1984), and thus each connection was expected to be comprised of distributed release sites on many branches. If this were to be true, differential electrotonic decay of the IPSPs from the different release sites of any one synapse to the recording electrode might have caused unequal increments in the probability distribution of peak amplitudes, or even too much apparent quantal variance for any peaks to be detectable (Redman, 1990). The present result therefore suggests either that the postsynaptic interneurons are electrotonically compact (which has been shown to be unlikely; Laurent, 1990), or that the release sites forming each synapse may generally be grouped and thus roughly equidistant from the recording site. Some quantal variability was nevertheless apparent (see below), which could possibly be explained by differential attenuation of quantal IPSPs from scattered release sites, and/or by intrinsic variability of quantal IPSPs.

The validity of a simple binomial and a Poisson model (DelCastillo and Katz, 1954; Boyd and Martin, 1956) was then tested on the experimental distribution of quanta. According to the simple binomial model, the probability $p(0<p<1)$ of release of one quantum at each of the $n$ available release sites is uniform and stationary, and the probability $p_{x}$ of observing $x$ quanta is given by

$$
p_{x}=\left\{\frac{n !}{(n-x) ! x !}\right\} p^{x}(1-p)^{n-x} .
$$

In the cases where $p$ is very small (i.e., where $m$ is very small when compared to $n$ ) the binomial distribution reduces to the Poisson distribution

$$
p_{x}=\frac{\left(e^{-m} m^{x}\right)}{x !}
$$

where $n$ and $p$ have no significance. Since quantal variability could not be measured directly from spontaneous potentials, some quantal variance $V_{q}=\sigma_{q}^{2}$ was assumed, to match the observed distribution (see Materials and Methods). A computer algorithm was then used to find the best fit to the observed distribution, using the $n, p$, and $\sigma_{q}$ (binomial) or $m$ and $\sigma_{q}$ (Poisson) as free parameters. For each connection, the average quantal content $m^{\prime}$ calculated from the product of $n$ and $p$ obtained from the best-fitting binomial theoretical distribution was compared to the value of $m$ determined indirectly as $I P S P / d V$ (see above and Table 1). Finally, $m^{\prime \prime}$ obtained from the coefficient of variation method (see Materials and Methods) was also compared to $m$ and $m^{\prime}$.

In 9 of the 10 connections analyzed in this way, the simple binomial model provided the better fit $(0.05$ significance level, $\chi^{2}$ test). The IPSP amplitude distribution histogram in Figure

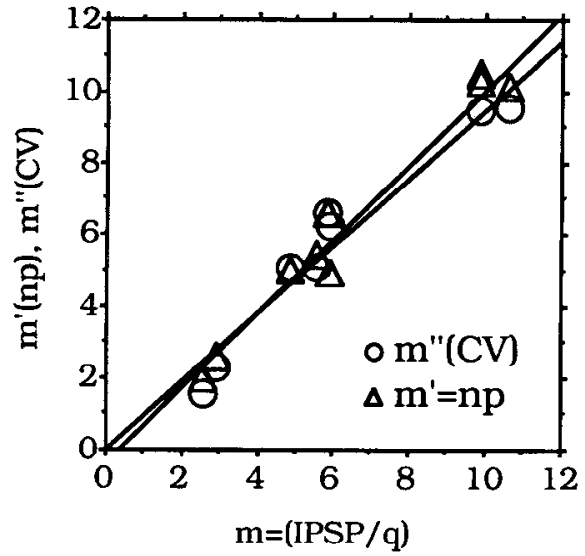

Figure 5. Scattergram illustrating the good correspondence between the values of quantal contents obtained independently by the three methods described in the text. Simple linear regression model; coefficients of determination, $0.97\left(m, m^{\prime}\right)$ and $0.96\left(m, m^{\prime \prime}\right)$.

4, for example, was best fitted with a theoretical distribution constructed with $n=13, p=0.367$, and $\sigma_{q}=120 \mu \mathrm{V}$ (Fig. 4C). Changing the bin size of the IPSP amplitude distribution histogram did not lead to significant changes in the best-fitting binomial parameters (Fig. $4 D$ ). The average size of the releasable pool $n$ for these nine connections was $13.1 \pm 2.8$ (ranging from 9 to 17 ), and the average release probability $p$ was $0.452 \pm 0.16$ (ranging from 0.2 to 0.68 ). Correspondence between the values of quantal contents $m, m^{\prime}$, and $m^{\prime \prime}$ estimated independently by the three methods was excellent $\left(0.01\right.$ significance level, $\chi^{2}$ test; see Tables 1, 2; Fig. 5).

\section{Simulation experiments: test of the significance of the histograms peaks and valleys}

One reasonable possibility was that both the finite number of PSP amplitude measurements and the data analysis procedures (e.g., arbitrary binning) had introduced misleading structure (false peaks and valleys) in the amplitude distribution histograms, and had thus affected our conclusions. If, for example, distribution histograms not significantly different from those obtained experimentally could be constructed from similar numbers of data points taken randomly from a smooth (i.e., nonquantized) distribution, our conclusion that each peak represents a quantal component would no longer be substantiated.

To address this possibility, we performed computer simulations of the following statistical experiment. We created sets of data points, drawn from a continuous smooth distribution that mimicked the behavior of the binomial distribution. This is as

Table 2. Comparisons of the values of mean quantal contents $m, m^{\prime}, m^{\prime \prime}$ obtained using the three independent methods described in Materials and Methods, for the two sets of connections detailed in

\begin{tabular}{|c|c|c|c|c|c|}
\hline & \multicolumn{3}{|c|}{ Pre 1 (Fig. 7) to: } & \multicolumn{2}{|c|}{ Pre 2 (Fig. 8) to: } \\
\hline & $\begin{array}{l}\text { Post } 1 \\
\text { (Fig. } 7 B \text { ) }\end{array}$ & $\begin{array}{l}\text { Post } 2 \\
\text { (Fig. } 7 C \text { ) }\end{array}$ & $\begin{array}{l}\text { Post } 3 \\
\text { (Fig. } 7 D \text { ) }\end{array}$ & $\begin{array}{l}\text { Post } 1 \\
\text { (Fig. } 8 A \text { ) }\end{array}$ & $\begin{array}{l}\text { Post } 2 \\
\text { (Fig. } 8 B \text { ) }\end{array}$ \\
\hline$m=I P S P / q$ & 5.530 & 6.000 & 10.590 & 2.580 & 9.880 \\
\hline$m^{\prime}=n p$ & 5.440 & 6.660 & 10.130 & 2.000 & 10.320 \\
\hline$m^{\prime \prime}(\mathrm{CV})$ & 5.070 & 6.640 & 9.600 & 1.580 & 9.510 \\
\hline
\end{tabular}
Figures 7 and 8

Pre and Post refer to pre- and postsynaptic neurons, respectively. 
A $\mathbf{X}$
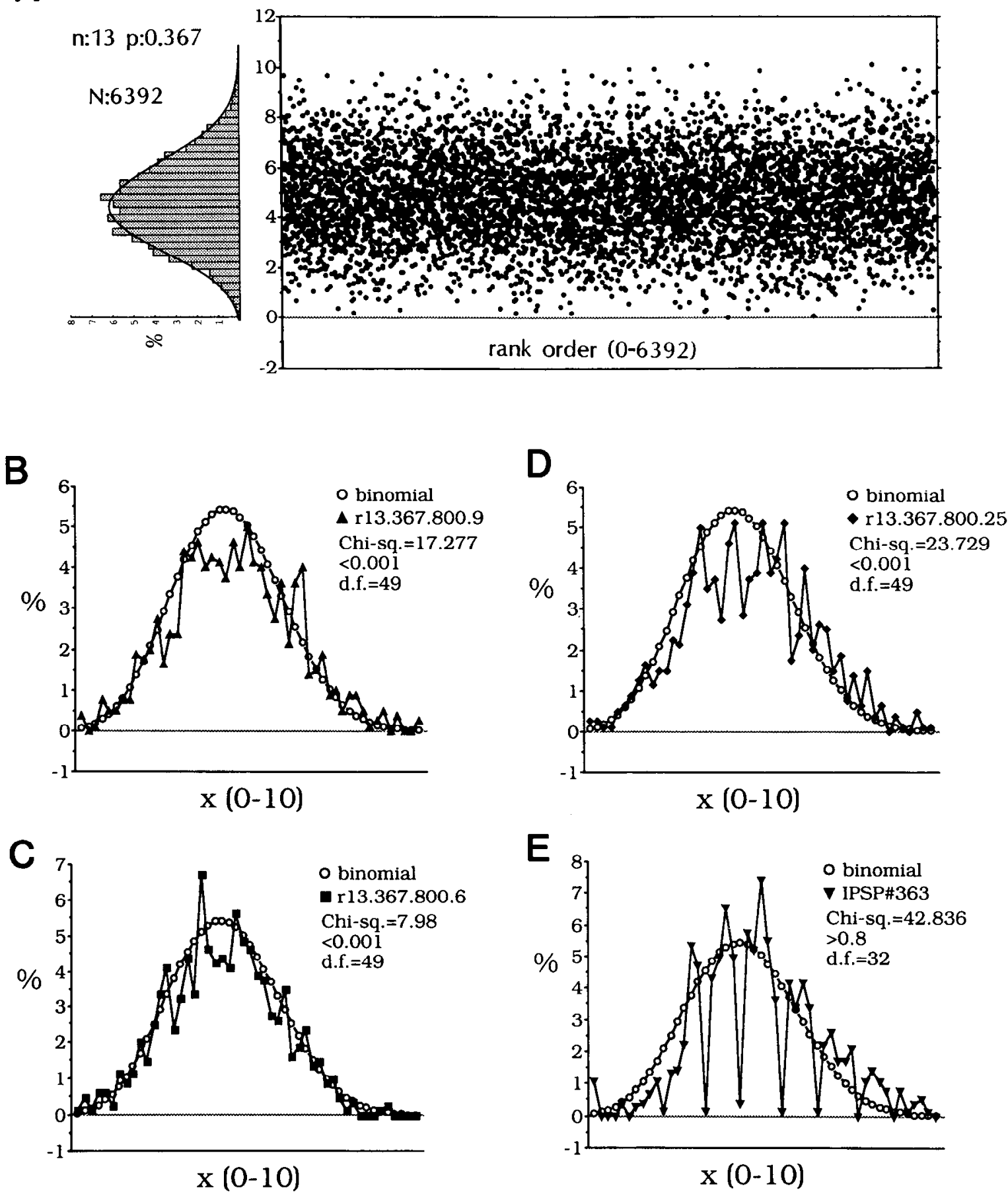

Figure 6. Computer simulation of a statistical experiment. A, Points (6392) were drawn randomly from a smooth binomial distribution (see text for methods), constructed with $n=13$ and $p=0.367$, that is, the values of the best fitting model for the experiment in Figure $4 C$. These data points were binned and represented in a frequency distribution histogram, which is expectedly well fitted by the "parent" theoretical distribution. $B-D$, Three computer simulation experiments $(r 13.367 .800 . i$, where $i$ is seed number), where only 800 data points were sampled, that is, a number similar to that of IPSP amplitude measurements $N$ in Figure 4. For each experiment, the frequency distribution of the values of $x$ (which in theory can vary from 0 to $n=13$ ) is represented against the "parent" theoretical curve. Data grouped here in 50 bins. A $\chi^{2}$ is then calculated to assess the goodness of fit between each simulation experiment and the theoretical curve. $d . f$., degrees of freedom. $E$, The theoretical curve is here plotted against the actual physiological experiment frequency distribution (IPSP \#363), and a $\chi^{2}$ calculated. The $\chi^{2}$ is calculated only with groups containing more than four data points, explaining the smaller number of degrees of freedom. The fit is worse than that for distributions in $B-D$, showing that the peaks and valleys of the histogram are unlikely to be due to limited sampling and arbitrary binning. 
close as one can get with a continuous distribution to the discrete binomial probability distribution. The binomial distribution for a given number of tries, $n$, and a given probability of the desired result on any particular try, $p$, is defined on the set of integers $\{0,1, \ldots, n\}$. We created a probability distribution that is a continuous function on the interval $[0, n]$ of the real line and has exactly the shape of the discrete binomial distribution.

This can be done by starting with the binomial distribution, which is given by

$$
B(j)=\frac{n !}{(n-j) ! j !} p^{j}(1-p)^{n-j},
$$

where $j$ is the number of successful results in an experiment of $n$ tries $(j$ in $\{0,1, \ldots, n\})$, and by noting the fact that the gamma function $(\Gamma)$, defined by

$$
\Gamma(z)=\int_{0}^{\infty} t^{z-1} e^{-t} d t,
$$

possesses the property

$$
\Gamma(n+1)=n !
$$

(Abramowitz and Stegun, 1964). A continuous function $G$ of the real variable $x$ can then be constructed by

$$
G(x)=\frac{\Gamma(n+1)}{\Gamma(n-x+1) \Gamma(x+1)} p^{x}(1-p)^{n-x}
$$

$G(j)$ is exactly equal to $B(j)$ at each integer $j$ in $\{0,1, \ldots, n\}$. A probability density function $H(x)$ on the sample space $[0, n]$ is created by normalizing $G$ :

$$
H(x)=K G(x)
$$

where

$$
K^{-1}=\int_{0}^{n} G(x) d x
$$

We then generated Monte Carlo numerical experiments for any desired $n$ and $p$ by using a random number generator and the method of rejection (Press et al., 1988) to create a realization of $N$ measurements of a random variable $x$, drawn from a sample with a probability density function $H(x)$. The random number generator could be seeded with different values, giving "independent experiments" with the same values of $n, p$, and $N$. One set of such numerical experiments is shown in Figure 6. The values of $n$ and $p$ were 13 and 0.367 , that is, identical to those used to fit the IPSP amplitude distribution histogram in the physiological experiment in Figure $4 C . N=6392$ data points were sampled randomly from the simulated distribution to generate the histogram in Figure $6 \mathrm{~A}$. This relatively smooth histogram was well fitted by the function $G$, as defined above (Eq. 7 ). When the sample size $N$ was decreased to values similar to those of the physiological experiments (here 800), frequency distributions such as those in Figure $6 B-D$ were generated. The goodness of fit between them and their "parent" theoretical distribution was then compared to that between the corresponding physiological experiment histogram (Fig. $6 E$ ) and the theoretical distribution. For each experiment, this procedure was repeated with three or four different bin sizes. In all studied cases, the goodness of fit between the theoretical curve and the distributions generated by the numerical experiments was highly significant (often at $<0.001$ significance level, $\chi^{2}$ ). The fit be-

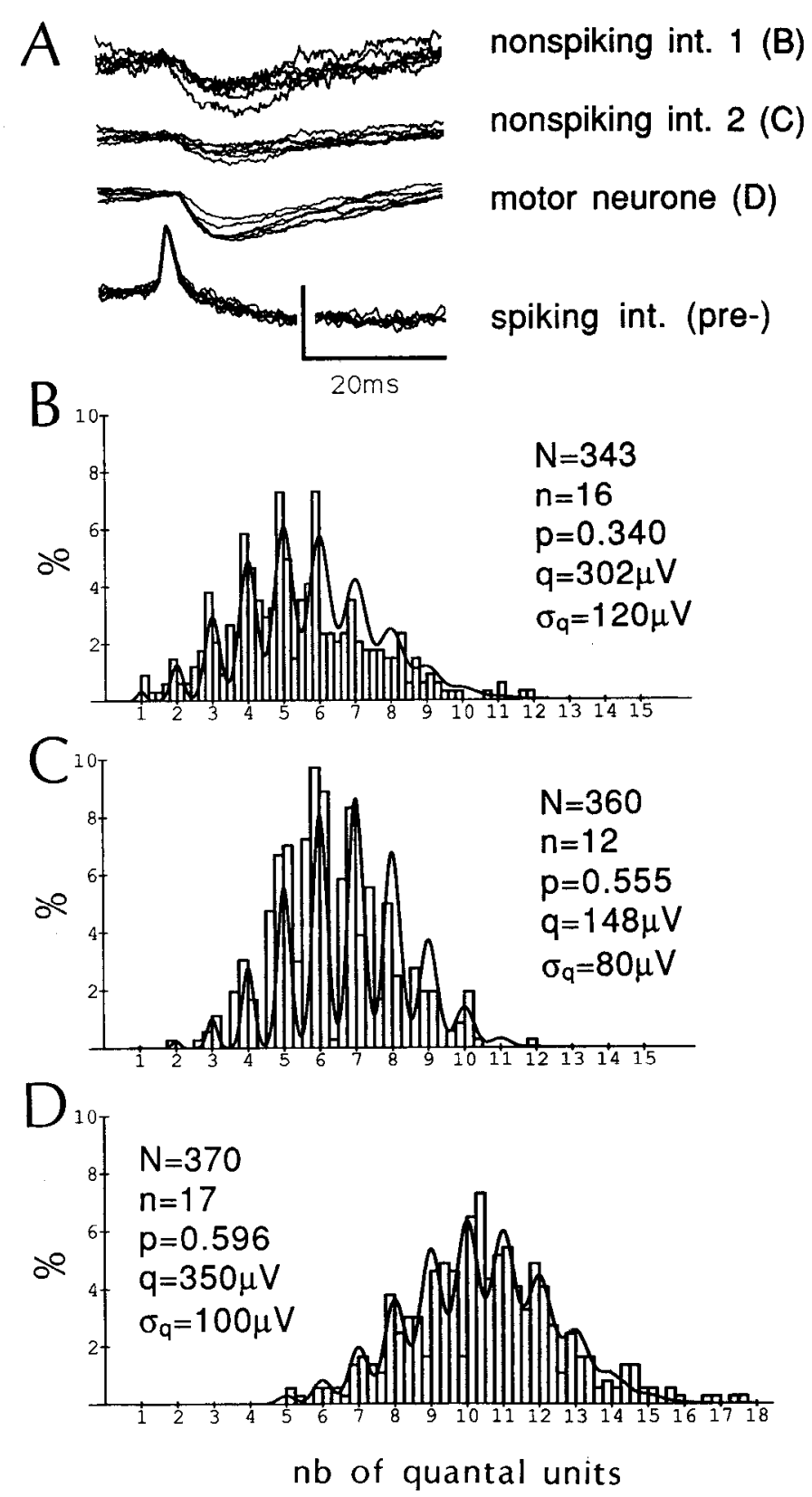

Figure 7. Quantal amplitude, quantal content, and binomials $n$ and $p$ differ between the synapses made by a single interneuron onto several targets. $A$, Spikes in the presynaptic local interneuron (bottom trace) evoke IPSPs in three target neurons recorded sequentially (top three traces). Calibration: $1 \mathrm{mV}$ (top trace), $2 \mathrm{mV}$ (2nd and 4th traces from top), $4 \mathrm{mV}$ (3rd from top). $B$ and $C$, IPSP amplitude distributions and best binomial fits for the two synapses made by the spiking interneuron in $A$ onto the two nonspiking interneurons. Note the differences in $n$, $p$, and $q$ between the two connections. $N$ is the number of IPSPs sampled. $D$, IPSP amplitude distributions and best binomial fit at the third synapse made by the same spiking interneuron onto a motorneuron, impaled in a neuropilar branch. The numbers of observed failures were 1 $(B$ and $C)$ and $2(D)$.

tween the theoretical curve and the physiological experiment distribution, however, was always poorer and never acceptable at the same significance levels. It should also be noticed that the quality of the fit was determined simply from bin-by-bin calculations; that is, no account was taken of the strong periodicity observed in the physiological data. The presence of such 
Figure 8. A spiking local interneuron that makes output synapses with two nonspiking local interneurons, with very different efficacies. Selected IPSPs evoked in the two nonspiking interneurons (post 1 and 2) by this spiking interneuron (pre) are shown in inset. The IPSP amplitude distribution histograms and the binomial fits for connections $l$ and 2 are shown in $A$ and $B$, respectively. Numbers of failures were $38(A)$ and $1(B)$. Calibration (inset): 1 $\mathrm{mV}$ (top and middle traces), $2 \mathrm{mV}$ (bottom trace); $12 \mathrm{msec}$.
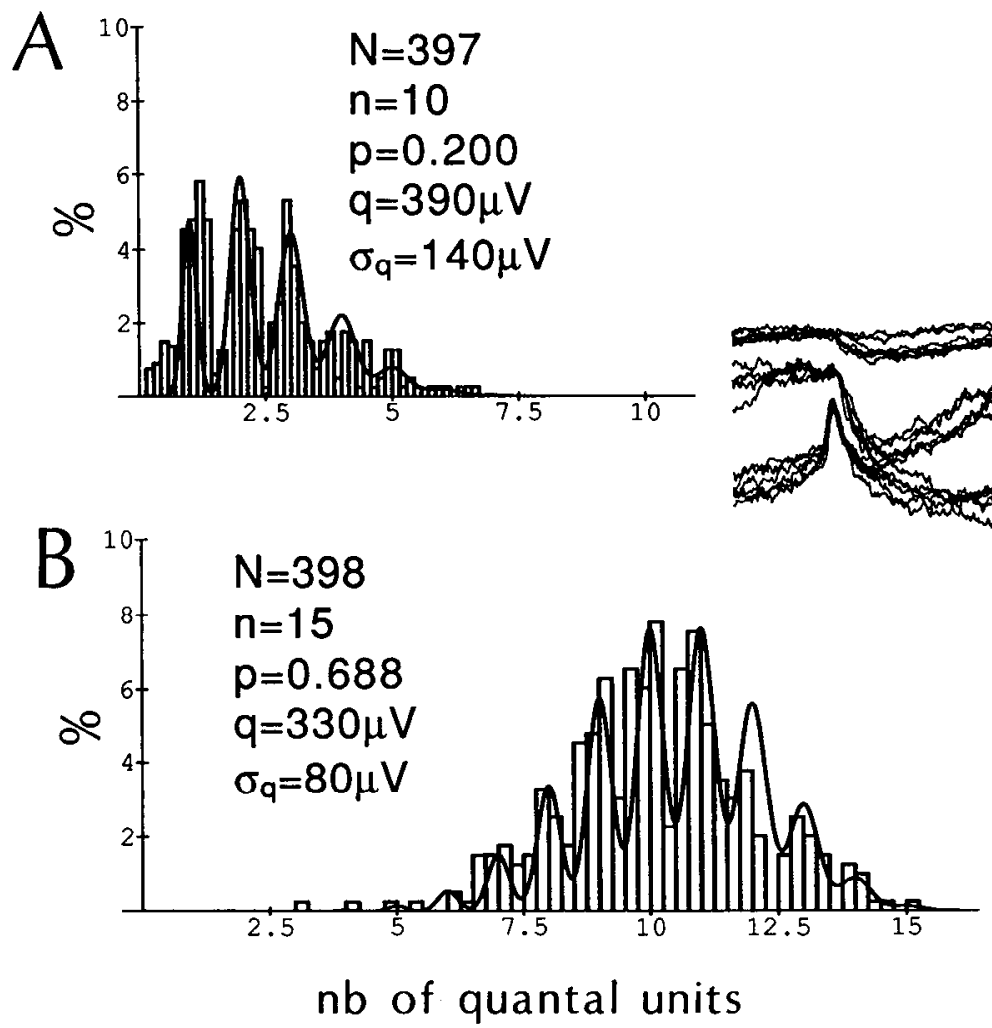

a feature would make the fit to the theoretical curve even worse, although it is hard to quantify by how much. These simulations therefore suggested that the structure of the IPSP amplitude histograms was indeed indicative of quantal release, and not an artifact or a consequence of the inherent limitations of the physiological experiments and binning procedures.

\section{Comparisons of release properties between synapses made by single interneurons onto different targets}

The binomial release parameters describing the synapses made by individual spiking interneurons onto different postsynaptic neurons were then compared. In Figure $7 A$, three connections made by a single spiking local interneuron onto two nonspiking local interneurons and one motorneuron sampled sequentially, and in identical conditions, are illustrated. Both binomial $n$ and $p$ (and also their product $m^{\prime}$ ) varied from synapse to synapse. At the synapse to the first nonspiking interneuron, best-fitting $n$ and $p$ were, respectively, 16 and 0.34 (Fig. $7 B$ ), but they were 12 and 0.55 at the synapse to the second nonspiking interneuron (Fig. $7 C$ ) and 17 and 0.59 at the synapse to the motorneuron (Fig. 7D). The mean quantal contents of these three different output synapses estimated from the three independent methods are given in Table 2. The quantal amplitudes at these three synapses were significantly distinct (Fig. 7), probably reflecting differences either in the electrotonic distance between release sites and electrode position, or in biophysical characteristics of the postsynaptic membranes, or both.

In Figure 8, a different spiking local interneuron showed even greater differences between two of its output synapses. At a synapse with one nonspiking interneuron, $n$ and $p$ were 10 and 0.2 , respectively, giving a mean quantal content of 2 (Fig. $8 \mathrm{~A}$ ). At a synapse with a second nonspiking interneuron, sampled $10 \mathrm{~min}$ later and identical conditions, $n$ and $p$ were 15 and 0.688 , respectively, with a mean quantal content of 10.32 (Fig.
$8 B)$. The corresponding mean quantal contents obtained at these two synapses using the three independent methods are indicated in Table 2.

\section{Discussion}

Synaptic transmission between spiking and nonspiking local interneurons in the locust CNS appears to be mediated by GABA. Pressure-applied GABA evokes an increase in membrane conductance in nonspiking interneurons, and a membrane polarization that reverses at the same apparent potential as the IPSC and IPSP evoked by action potentials from presynaptic spiking local interneurons. The present results thus support the immunocytochemical evidence that spiking local interneurons in the midline group show GABA-like immunoreactivity (Watson and Burrows, 1987). IPSPs had an average amplitude of 1.93 $\mathrm{mV}$, and the signal-to-noise ratio was high enough to allow the detection of distinct quantal peaks separated by an average of $290 \mu \mathrm{V}$. Conversely, the amplitude of the IPSPs was small enough, when compared with the driving force at this synapse, to prevent or reduce the nonlinear summation of quantal PSPs and thereby allow quantal analysis to be performed without correction. Quantal contents determined indirectly from the values of IPSP and quantal amplitudes matched closely those calculated from the best-fitting binomial parameters $n$ and $p$. Unfortunately, no ultrastructural evidence exists as yet on the numbers of morphological contacts between connected spiking and nonspiking local interneurons. It is therefore not possible to compare, for each established connection, the numbers of putative contacts to binomial $n$, as was done, for example, in the goldfish Mauthner cell (Korn et al., 1982), or to provide an estimate of the number of release sites per presynaptic varicosity.

The main result of this study, however, is that the statistical properties of release at the central synapses made by a single 
interncuron onto different target interneurons may differ, cven when the postsynaptic potentials are sampled in identical conditions of presynaptic activity. First, quantal contents can be different for the synapses made by single neurons onto different target neurons. Whether these observed differences arise as the result of developmental and/or plastic phenomena is so far unknown but will warrant further investigation. Differences in release properties, similar to those presented here, have been described in the PNS of crayfish, at the junctions made by a motor axon onto several muscle fibers (Atwood, 1967; Atwood and Bittner, 1971). There, the differences in size of the evoked synaptic currents between junctions are correlated with differences in the ability of each junction to facilitate. Synapses with low quantal contents tend to facilitate more than those with high quantal contents. Experiments to test this possibility at the present central synapse have not yet been conducted. The reported results, however, may have gencral implications for the study of synaptic plasticity in the CNS. If, for example, plastic phenomena such as long-term potentiation in mammalian hippocampus involve presynaptic modifications (Bekkers and Stevens, 1990; Malinow and Tsien, 1990; Malinow, 1991), it will be crucial to determine if, for a given neuron, such presynaptic changes affect only those synapses that were specifically reinforced (by simultaneous pre- and postsynaptic depolarization), or if they affect all or many of the synpases made by the particular neuron that is undergoing plastic changes. Results from rat hippocampus slice cultures indeed suggest that the pairing of pre- and postsynaptic stimulation leads to distributed synaptic strengthening (Bonhoeffer et al., 1989). In addition, theoreticians often assume that individual synapses made by a presynaptic neuron can be modified without altering the efficacy of other synapses made by the same neuron. Insofar as the plastic changes are due to presynaptic modifications, the capability for synapse-specific change would require that different terminals of a given presynaptic neuron have different release properties. The present work helps establish that this is possible.

Second, it is suggested that these differences in quantal contents between synapses are the result of differences both in the size of the releasable pool (corresponding possibly to the number of release sites) of each synapse, and in the probability of quantal release at each synapse. This conclusion is model dependent. A simple binomial model of transmitter release was used here, and one of its main assumptions is that the probability of release $p$ is uniform at all the sites forming one functional synapse with a particular neuron (McLachlan, 1978; Redman, 1990). This would therefore imply that the probability of release $p$ is here uniform among the sites forming one synapse, but that it differs between the release sites associated with different postsynaptic neurons. Clustering of the release sites in contact with the same postsynaptic neuron, so that the amplitude and duration of the presynaptic depolarization due to spike invasion and $\left[\mathrm{Ca}^{2+}\right]_{i}$ are similar at all these sites, might contribute to this uniformity of $p$. Different distributions of $\mathrm{K}^{+}$channels on the branches forming different synapses, by contrast, could shape passively invading spikes differently and thus contribute to the nonuniformity of $p$ between synapses to different neurons. The validity of the assumption that $p$ is uniform for each synapse could not be verified. A compound binomial model, which would relax the condition of uniformity (Walmsley et al., 1987), has not been tested, and it is therefore not known whether it would provide a better fit to the data. It seems, however, that, whichever of these two binomial models best describes the experi- mental data, release probabilities at all the central release sites of single locust spiking local interneurons will have to be nonuniform, to account for the present results.

Finally, these results suggest that the integrative unit in these local circuits may often be the synapse rather than the entire interneuron. Indeed, the ability to detect distinct peaks in the IPSP amplitude distribution histograms from interneurons with such complex electrical geometry (Laurent, 1990, 1991) suggests that the anatomical contacts forming each functional synapse were at similar electrotonic distances from the recording site and were therefore possibly clustered. Since input and output synapses are intermingled on the branches of the nonspiking interneurons (Watson and Burrows, 1988), synaptic inputs originating from one presynaptic spiking local interneuron might affect only the neighboring nonspiking output sites but not those that are electrotonically distant. Single branches or dendritic rcgions of the nonspiking interneurons might thus opcrate as local input-output units. The present results thus support other physiological evidence that indicates that nonspiking local interneurons may be functionally compartmentalized (Laurent and Burrows, 1989; Laurent, 1990, 1991).

Note added in proof: A recent article by Gardner(1991) describes experiments in Aplysia buccal ganglia, showing that synaptic current amplitudes are different for different synapses made by a single neuron on different targets. Gardner used the coefficient of variation method of quantal analysis to show that these differences in current amplitude are due to presynaptic factors. This result is thus in agreement with our own. Gardner also reports that the statistical properties of release of several "fibers" that originate from different neurons, but converge on the same target, are similar. Although we did not study this configuration systematically, we did compare the properties of release of two neurons converging on a single target in one occasion. The release properties of these two neurons were significantly different from each other.

\section{References}

Abramowitz M, Stegun IA (1964) Applied mathematics series, Vol 55 , I Iandbook of mathematical functions. Washington, DC: National Bureau of Standards.

Atwood HL (1967) Variation in physiological properties of crustacean motor synapses. Nature 215:57-58.

Atwood HL, Bittner GD (1971) Matching of excitatory and inhibitory inputs to crustacean muscle fibers. J Neurophysiol 34:157-170.

Bacon JP, Altman JS (1977) A silver intensification method for cobaltfilled neurons in whole mount preparation. Brain Res 138:359-363.

Bekkers J, Stevens C (1990) Presynaptic mechanism for long-term potentiation in the hippocampus. Nature 346:724-729.

Bonhoeffer T, Staiger V, Aertsen A (1989) Synaptic plasticity in rat hippocampal slice cultures: local "Hebbian" conjunction of pre- and postsynaptic stimulation leads to distributed synaptic enhancement. Proc Natl Acad Sci USA 86:8113-8117.

Boyd IA, Martin AR (1956) The end-plate potential in mammalian muscle. J Physiol (Lond) 132:74-91.

Burrows M (1980) The control of sets of motor neurons by local interneurones in the locust. J Physiol (Lond) 298:213-233.

Burrows M (1987a) Parallel processing of proprioceptive signals by spiking local interneurons and motor neurons in the locust. J Neurosci 7:1064-1080.

Burrows M (1987b) Inhibitory interactions between spiking and nonspiking local interneurons in the locust. J Neurosci 7:3282-3292.

Burrows M, Siegler MVS (1978) Graded synaptic transmission between local interneurones and motor neurones in the metathoracic ganglion of the locust. J Physiol (Lond) 285:231-255.

Burrows M, Siegler MVS (1982) Spiking local interneurons mediate local reflexes. Science 217:650-652. 
DelCastillo J, Katz B (1954) Quantal components of the endplate potential. J Physiol (Lond) 124:560-573.

Edwards FA, Konnerth A, Sakmann B (1990) Quantal analysis of inhibitory synaptic transmission in the dentate gyrus of rat hippocampal slices-a patch-clamp study. J Physiol (Lond) 430:213-249.

Faber DS, Korn H (1988) Unitary conductance changes at teleost Mauthner cell glycinergic synapses - a voltage-clamp and pharmacologic analysis. J Neurophysiol 60:1982-1999.

Freund TF, Martin KAC, Whitteridge D (1985) Innervation of cat visual areas 17 and 18 by physiologically identified X-and Y-type thalamic afferents. I. Arborization patterns and quantitative distribution of postsynaptic elements. J Comp Neurol 242:263-274.

Gardner D (1991) Presynaptic transmitter release is specified by postsynaptic neurons of Aplysia buccal ganglia. J Neurophysiol 66:21502154.

Korn H, Mallet A, Triller A, Faber DS (1982) Transmission at a central synapse. II. Quantal description of release, with a physical correlate for binomial $n$. J Neurophysiol 48:679-707.

Larkman A, Stratford K, Jack J (1990) Quantal analysis of excitatory synaptic action and depression in hippocampal slices. Nature 350 : 344-347.

Laurent $G$ (1987) The role of spiking local interneurons in shaping the receptive fields of intersegmental interneurons in the locust. $J$ Neurosci 7:2977-2989.

Laurent G (1990) Voltage-dependent nonlinearities in the membrane of locust nonspiking local interneurons, and their significance for synaptic integration. J Neurosci 10:2268-2280.

Laurent $G$ (1991) Evidence for voltage-activated outward currents in the neuropilar membrane of locust nonspiking local interneurons. J Neurosci 11:1713-1726.

Laurent G, Burrows M (1989) Intersegmental interneurons can control the gain of reflexes in adjacent segments of the locust by their action on nonspiking local interneurons. J Neurosci 9:3030-3039.

Laurent $G$, Hustert R (1988) Motor neuronal receptive-field delimit patterns of motor activity during locomotion of the locust. J Neurosci $8: 4349-4366$.

Llinás RR, Walton KD (1990) Cerebellum. In: The synaptic organization of the brain (Shepherd GM, ed), pp 214-245. New York: Oxford UP.
Malinow R (1991) Transmission between pairs of hippocampal slice neurons-quantal levels, oscillations, and LTP. Science 252:722-724.

Malinow R, Tsien RW (1990) Presynaptic enhancement shown by whole-cell recordings of long-term potentiation in hippocampal slices. Nature 346:177-180.

McLachlan EM (1978) The statistics of transmitter release at chemical synapses. Int Rev Physiol Neurophysiol III 17:49-117.

Nagayama T, Burrows M (1990) Input and output connections of an anteromedial group of spiking local interneurons in the metathoracic ganglion of the locust. J Neurosci 10:785-794.

Pitman RM, Tweedle CD, Cohen MI (1972) Branching of central neurones: intracellular cobalt injection for light and electron microscopy. Science 176:412-414.

Press WH, Flannery BP, Teukolsky SA, Vetterling WY (1988) Numerical recipes in C. Cambridge, MA: Cambridge UP.

Redman S (1990) Quantal analysis of synaptic potentials in neurons of the central nervous system. Physiol Rev 70:165-198.

Siegler MVS, Burrows M (1983) Spiking local interneurones as primary integrators of mechanosensory information in the locust. J Neurophysiol 50:1281 -1295.

Siegler MVS, Burrows M (1979) Morphology of local non-spiking interneurons in the metathoracic ganglion of the locust. J Comp Neurol 183:121-148.

Siegler MVS, Burrows M (1984) The morphology of two groups of spiking local interneurones in the metathoracic ganglion in the locust. J Comp Neurol 224:463-482.

Walmsley B, Edwards FR, Tracey DJ (1987) The probabilistic nature of synaptic transmission at a mammalian excitatory central synapse. J Neurosci 7:1037-1046.

Watson AHD, Burrows M (1985) The distribution of synapses on the two fields of neurites of spiking local interneurons in the locust. $J$ Comp Neurol 240:219-232.

Watson AHD, Burrows M (1987) Immunocytochemical and pharmacological evidence for GABA-ergic spiking local interneurons in the locust. J Neurosci 7:1741-1751.

Watson AHD, Burrows M (1988) Distribution and morphology of synapses on nonspiking local interneurones in the thoracic nervous system of the locust. J Comp Neurol 272:605-616. 